\title{
ESTIMATES OF THE INTEGRAL REMAINDERS IN SEVERAL NUMERICAL INTEGRAL FORMULAS USING THE HENSTOCK-KURZWEIL INTEGRAL
}

\author{
Xiaofeng Ding, Guoju Ye and Wei-Chi Yang
}

Abstract. Some integral remainders of Trapezoidal, Corrective Trapezoidal and Simpson formulas are given. The Hölder inequality and Henstock-Kurzweil integral are used to estimate the remainders in terms of Alexiewicz and Lebesgue $p$-norms.

Mathematics subject classification (2000): Primary 28B05, 46G10 ; Secondary 26A39.

Keywords and phrases: integral remainder, Henstock-Kurzweil integral, Hölder inequality, Alexiewicz norm.

\section{REFERENCES}

[1] S. S. DRAGOMIR, New Estimate of The Remainder in Taylor's Formula Using Grüss's Type Inequalities and Applications, Math. Ineq. Appl 2 , No. 2 (1999), 183-193.

[2] H. B. Thompson, Taylor's Theorem Using The Geralized Riemann Integral, Amer. Math. Monthly, 96 (1989), 346-350.

[3] ERIK Talvila, Estimates of The Remainder in Taylor's Theorem Using The Henstock-Kurzweil Integral, Czechoslovak Mathmatical Joural 55 (130) (2005), 933-940.

[4] Lee Peng-Yee and Rudolf Vyborny, The Integral: An Easy Approach after Kurzweil and Henstock, Cambridge University Press, Cambridge, UK, 2000.

[5] LeE PENG-YEE, Lanzhou Lectures on Henstock Integration, World Scientific, Singapore, 1989.

[6] R. A. Gordon, The Integral of Lebesgue, Denjoy, Perron, and Henstock, Graduate Studies in Mathematics, Amer. Math. Soc., 1994.

[7] Wang Lie-heng, Residuals For Some Quadratures in Integral Form, Mathematics in Practice and Theory No. (3) (1990), 23-28.

[8] P. DaVis And P. Rabinowitz, Methods of Numerical Integration, Academic Press, New York,1975.

[9] Wei-Chi Yang and Jonathan Lewin, Exploring Mathematics With Scientific Notebook, Springer, 2002.

[10] S. SchwabiK AND Guoju Ye, Topics in Banach Space Integration, World Scientific, 2005. 